$\xi$

\title{
On double stage minimax-shrinkage estimator for generalized Rayleigh model
}

\author{
Abbas Najim Salman *, Maymona M. Ameen \\ Department of Mathematics-Ibn-Al-Haitham College of Education - University of Baghdad \\ *Corresponding authorE-mail: abbasnajim66@yahoo.com
}

\begin{abstract}
This paper is concerned with minimax shrinkage estimator using double stage shrinkage technique for lowering the mean squared error, intended for estimate the shape parameter $(\alpha)$ of Generalized Rayleigh distribution in a region (R) around available prior knowledge $\left(\alpha_{0}\right)$ about the actual value $(\alpha)$ as initial estimate in case when the scale parameter $(\lambda)$ is known .

In situation where the experimentations are time consuming or very costly, a double stage procedure can be used to reduce the expected sample size needed to obtain the estimator.

The proposed estimator is shown to have smaller mean squared error for certain choice of the shrinkage weight factor $\psi(\cdot)$ and suitable region $\mathrm{R}$.

Expressions for Bias, Mean squared error (MSE), Expected sample size [E (n/ $\alpha, \mathrm{R})]$, Expected sample size proportion $[\mathrm{E}(\mathrm{n} / \alpha, \mathrm{R}) / \mathrm{n}]$, probability for avoiding the second sample $\left[\mathrm{p}\left(\underset{1}{\hat{\alpha} \in \mathrm{R})]}\right.\right.$ and percentage of overall sample saved $\left.{ }_{\left[\frac{\mathrm{n}_{2}}{\mathrm{n}}\right.} \mathrm{p}(\underset{1}{\hat{\alpha}} \in \mathrm{R}) * 100\right]$ for the proposed esti-
\end{abstract} mator are derived.

Numerical results and conclusions for the expressions mentioned above were displayed when the consider estimator are testimator of level of significance $\Delta$.

Comparisons with the minimax estimator and with the most recent studies were made to shown the effectiveness of the proposed estimator.

Keywords: Generalized Rayleigh Distribution, Maximum Likelihood Estimator, Minimax Estimator, Double Stage Shrinkage Estimator, Mean Squared Error and Relative Efficiency.

\section{Introduction}

Burr [4] introduced different forms of cumulative distribution functions for modeling lifetime data. Among those distributions, Burr Type X and Burr Type XII are the most popular ones. Several authors considered different aspects of the Burr Type $X$ and Burr Type XII distribution, see for example [12], [14] and [19]. Also, Burr Type X has been studied by [3] and [13].

Two parameters Burr Type $X$ distribution and correctly named as two parameter Generalized Rayleigh distribution are introduced in [15] and [16]. They showed that the two parameters Generalized Rayleigh distribution can be used quite effectively in modeling strength data and also in modeling general life time data. Different estimators are considered in [7] and they studied how the estimator of the different unknown parameter behaves for different sample size.

The two parameters Generalized Rayleigh (GR) distribution has the following distribution function:

$$
\mathrm{F}(\mathrm{x} ; \alpha, \lambda)=\left[1-\mathrm{e}^{-(\lambda \mathrm{x})^{2}}\right]^{\alpha} \text { for } \mathrm{x}>0, \alpha>0, \lambda>0
$$

Thus, the probability density function (p.d.f.) of (GR) distribution is

$$
f(x ; \alpha, \lambda)=\left\{\begin{array}{lr}
2 \alpha \lambda^{2} x e^{-(\lambda x)^{2}}\left(1-e^{-(\lambda x)^{2}}\right)^{\alpha-1} & \text { for } x>0, \alpha, \lambda>0 \\
0 & \text { o.w. }
\end{array}\right.
$$

Where, $\alpha$ and $\lambda$ are the shape and scale parameters respectively.

A double stage shrinkage estimator procedure has the following steps:

Let $\mathrm{x}_{1 \mathrm{i}} ; \mathrm{i}=1,2 \ldots \mathrm{n}_{1}$ be a random sample of $\mathrm{n}_{1}$ from GR distribution and $\hat{\alpha}_{1}$ be a "good" estimator of $\alpha$ based on these $n_{1}$ observation. Construct a preliminary test region $\mathrm{R}$ in the parameter space $\alpha$ based on $\alpha_{0}$ and an appropriate criterion for test statistic.

If $\hat{\alpha}_{1} \in \mathrm{R}$, shrink $\hat{\alpha}_{1}$ towards $\alpha_{0}$ by shrinkage weight factor $0 \leq \psi(\hat{\alpha}) \leq 1$ and use the shrinkage estimator $\psi\left(\hat{\alpha}_{1}\right) \hat{\alpha}_{1}+\left(1-\psi\left(\hat{\alpha}_{1}\right)\right) \alpha_{0}$, for estimate $\alpha$.

If $\hat{\alpha}_{1} \notin R$, obtain $x_{2 i} ; i=1,2 \ldots n_{2}$, an additional sample of size $n_{2}$ and use a pooled estimator $\hat{\alpha}_{p}$ of $\alpha$ based on combined samples of size $\mathrm{n}=\mathrm{n}_{1}+\mathrm{n}_{2}$, i.e.; $\hat{\alpha}_{\mathrm{p}}=\frac{\mathrm{n}_{1} \hat{\alpha}_{1}+\mathrm{n}_{2} \hat{\alpha}_{2}}{\mathrm{n}}$.

Thus, the double stage shrinkage estimator (DSSE) of $\alpha$ will be:

$\widetilde{\alpha}_{D S}=\left\{\begin{array}{c}\Psi_{1}\left(\widehat{\alpha}_{1}\right) \widehat{\alpha}_{1}+\left(1-\Psi_{1}\left(\widehat{\alpha}_{1}\right)\right) \alpha_{0}, \text { if } \widehat{\alpha}_{1} \in R \\ \widehat{\alpha}_{\mathrm{p}}, \text { if } \widehat{\alpha}_{1} \notin R\end{array}\right.$ 
The motivation of this study was provided by the work of [1], [2], [6], [9] and [18].

The minimax estimation is an upgraded non-classical approach in the estimated area of statistical inference, which was introduced by Abraham Wald (1945) from the concept of game theory. It opens a new dimension in statistical estimation and enriched the method of point estimations. Von Neumann [1944] introduced the word minimax in game theory which is the optimum strategy of the second player in the two person zero game. According to Abraham Wald, "minimax approach tries to guard against the worst by requiring that the chosen decision rule should provide maximum protection against the highest possible risk". An estimator having this property is called a minimax estimator. The most important elements in the minimax approach are the specification of the prior distribution and the loss function used. In this paper, modified linear exponential (MLINEX) loss functions have been used to obtain the minimax estimators of the parameter of the Generalized Rayleigh distribution, [5], [8], [10] and [11].

The aim of this paper is to create the double stage shrinkage estimator (DSSE) $\widetilde{\alpha}_{D S}$ defined by (3) during employ the minimax estimator $\left(\widehat{\alpha}_{\mathrm{B} 1}=\widehat{\alpha}_{\mathrm{MML}}\right)$ instead of the classical estimator (MLE) $\widehat{\alpha}_{1}$ for estimate the shape parameter $(\alpha)$ of two parameters Generalized Rayleigh (GR) distribution when the scale parameter $(\lambda)$ is known.

The expressions of Bias, Mean squared error (MSE), Relative Efficiency [R.Eff(.)], Expected sample size, Expected sample size proportion, probability for avoiding the second sample and percentage of overall sample saved are derived and obtained for the proposed estimator $\widetilde{\alpha}_{D S}$.

Numerical results and conclusions due mentioned expressions including some constants are performed and displayed in annexed tables.

Comparisons between the proposed estimator with the minimax estimator $\widehat{\alpha}_{\mathrm{B} 1}$ and with some of the last studies are demonstrated.

\section{Minimax shrinkage estimator of $\alpha$}

In this section, we obtain the minimax estimators of the parameter $\alpha$ for the Burr-X distribution. The derivation depends primarily on a theorem, which is due to Hodge and Lehmann (1950), [5], [8], [10] and [11], and can be stated as follows:

Lehmann's Theorem: If $\tau=\left\{F_{\alpha} ; \alpha \in \Theta\right\}$ be a family of distribution functions and $\mathrm{D}$ a class of estimators of $\alpha$. Suppose that $\mathrm{d}^{*} \in \mathrm{D}$ is a Bayes estimator against a prior distribution $\xi^{*}(\alpha)$ on the parameter space $\Theta$, and the risk function $R\left(d^{*}, \alpha\right)$ constant on $\Theta$; then $\mathrm{d}^{*}$ is a minimax estimator of $\alpha$.

\section{Main results}

Theorem: Let $\mathrm{X}_{1}, \mathrm{X}_{2}, \ldots, \mathrm{X}_{\mathrm{n}}$ be $n$ independently and identically distributed random variables drawn from the density (2), then $d^{*}=\hat{\alpha}_{M M L}=\left[\frac{\Gamma(n)}{\Gamma(n-c)}\right]^{\frac{1}{c}} / \sum_{i=1}^{n} \ln \left(1-e^{-\left(\lambda x_{i}\right)^{2}}\right)^{-1}$ is the minimax estimator $\hat{\alpha}_{M M L}$ of the parameter $\alpha$ for the MLINEX loss function of the type,

$\mathrm{L}\left(\alpha, \mathrm{d}_{2}\right)=\omega\left[\left(\frac{\widehat{\alpha}_{\mathrm{B}}}{\alpha}\right)^{\mathrm{c}}-\operatorname{cln}\left(\frac{\widehat{\alpha}_{\mathrm{B}}}{\alpha}\right)-1\right] ; \omega>0, \mathrm{c} \neq 0$

Where $\mathrm{d}^{*}$ is the estimate of $\alpha, \omega$ and $\mathrm{c}$ are two known parameters the loss function.

Proof: to prove the theorem we have to use Lehmann's theorem, in order to prove the theorem it will be sufficient to show that $\mathrm{d}^{*}=\widehat{\alpha}_{\mathrm{B}}=\left[\frac{\Gamma(\mathrm{n})}{\Gamma(\mathrm{n}-\mathrm{c})}\right]^{\frac{1}{\mathrm{c}}} \frac{1}{\sum_{\mathrm{i}=1}^{\mathrm{n}} \ln \left(1-\mathrm{e}^{-\left(\lambda \mathrm{x}_{\mathrm{i}}\right)^{2}}\right)^{-1}}$ is a minimax estimator $\widehat{\alpha}_{\text {MML }}$ of $\alpha$ for the loss function (4). For this, first we have to find the Bayes estimator $\mathrm{d}^{*}$ of $\alpha$. Then if we can show that the risk of $d^{*}$ is constant, then the theorem will be proved. As before, using the non-informative prior, we get the posterior distribution of $\alpha$ as:

$$
\begin{aligned}
& \mathrm{g}\left(\alpha \mid \mathrm{x}_{1}, \mathrm{x}_{2}, \ldots, \mathrm{x}_{\mathrm{n}}\right)=\frac{\left[\prod_{i=1}^{\mathrm{n}} \mathrm{f}(\mathrm{x} ; \alpha)\right] \mathrm{g}(\alpha)}{\int_{\alpha}\left[\prod_{\mathrm{i}=1}^{\mathrm{n}} \mathrm{f}(\mathrm{x} ; \alpha)\right] \mathrm{g}(\alpha) \mathrm{d} \alpha} \\
& \therefore \mathrm{g}\left(\alpha \mid \mathrm{x}_{1}, \mathrm{x}_{2}, \ldots \mathrm{x}_{\mathrm{n}}\right)= \\
& \frac{\alpha^{\mathrm{n}-1}}{\Gamma(\mathrm{n})}\left(\sum_{\mathrm{i}=1}^{\mathrm{n}} \ln \left(1-\mathrm{e}^{-\left(\lambda \mathrm{x}_{\mathrm{i}}\right)^{2}}\right)^{-1}\right)^{\mathrm{n}} \mathrm{e}^{-\alpha \sum_{\mathrm{i}=1}^{\mathrm{n}} \ln \left(1-\mathrm{e}^{-\left(\lambda \mathrm{x}_{\mathrm{i}}\right)^{2}}\right)^{-1}} ; \alpha>, \lambda>0, \mathrm{x}> \\
& 0
\end{aligned}
$$

Now the Bayes estimator of $\alpha$ under the MLINEX loss function (4) is:

$\mathrm{d}^{*}=\widehat{\alpha}_{\mathrm{BML}}=\left[\mathrm{E}_{\alpha}\left(\alpha^{-\mathrm{c}}\right)\right]^{-\frac{1}{\mathrm{c}}}$

Where

$E_{\alpha}\left(\alpha^{-c}\right)=\int_{\alpha} \alpha^{-c} g(\alpha \mid x) d \alpha$

$=\frac{\left(\sum_{\mathrm{i}=1}^{\mathrm{n}}\left(1-\mathrm{e}^{-\left(\lambda \mathrm{x}_{\mathrm{i}}\right)^{2}}\right)^{-1}\right)^{\mathrm{n}}}{\Gamma(\mathrm{n})} \int_{0}^{\infty} \alpha^{-\mathrm{c}} \mathrm{e}^{-\alpha\left(1-\mathrm{e}^{-\left(\lambda \mathrm{x}_{\mathrm{i}}\right)^{2}}\right)^{-1}} \alpha^{\mathrm{n}-1} \mathrm{~d} \alpha$

$=\frac{\left(\sum_{\mathrm{i}=1}^{\mathrm{n}}\left(1-\mathrm{e}^{-\left(\lambda \mathrm{x}_{\mathrm{i}}\right)^{2}}\right)^{-1}\right)}{\Gamma(\mathrm{n})} \cdot \frac{\Gamma(\mathrm{n}-\mathrm{c})}{\left(\sum_{\mathrm{i}=1}^{\mathrm{n}}\left(1-\mathrm{e}^{-\left(\lambda x_{\mathrm{i}}\right)^{2}}\right)^{-1}\right)^{\mathrm{n}-\mathrm{c}}}$

$=\frac{\Gamma(\mathrm{n}-\mathrm{c})}{\Gamma(\mathrm{n})} \cdot\left(\sum_{\mathrm{i}=1}^{\mathrm{n}}\left(1-\mathrm{e}^{-\left(\lambda \mathrm{x}_{\mathrm{i}}\right)^{2}}\right)^{-1}\right)^{\mathrm{c}}$

Using this result we get

$\mathrm{d}^{*}=\widehat{\alpha}_{\mathrm{BML}}=\left[\frac{\Gamma(\mathrm{n}-\mathrm{c})}{\Gamma(\mathrm{n})} \cdot\left(\sum_{\mathrm{i}=1}^{\mathrm{n}}\left(1-\mathrm{e}^{-\left(\lambda \mathrm{x}_{\mathrm{i}}\right)^{2}}\right)^{-1}\right)^{\mathrm{c}}\right]^{-1 / \mathrm{c}}=$

$\left[\frac{\Gamma(\mathrm{n}-\mathrm{c})}{\Gamma(\mathrm{n})}\right]^{-1 / \mathrm{c}} \frac{1}{\sum_{\mathrm{i}=1}^{\mathrm{n}} \ln \left(1-\mathrm{e}^{-\left(\lambda x_{\mathrm{i}}\right)^{2}}\right)^{-1}}=\frac{\mathrm{K}}{\mathrm{T}}$

Where $\mathrm{K}=\left[\frac{\Gamma(\mathrm{n}-\mathrm{c})}{\Gamma(\mathrm{n})}\right]^{-1 / \mathrm{c}}$ and $\mathrm{T}=\ln \sum_{\mathrm{i}=1}^{\mathrm{n}}\left(1-\mathrm{e}^{-\left(\lambda \mathrm{x}_{\mathrm{i}}\right)^{2}}\right)^{-1}$ is the complete sufficient statistic for $\alpha$.

Now the risk function under the MLINEX (4) is given by

$R_{M}(\alpha)=E\left[L\left(\alpha, \hat{\alpha}_{B M L}\right)\right]$

$=\omega E\left[\left(\frac{\widehat{\alpha}_{B M L}}{\alpha}\right)^{c}-\operatorname{cln}\left(\frac{\widehat{\alpha}_{B M L}}{\alpha}\right)-1\right.$

$=\omega\left[\frac{1}{\alpha^{c}} E\left(\hat{\alpha}_{B M L}{ }^{c}\right)-c E\left(\ln \hat{\alpha}_{B M L}\right)+c \ln \alpha-1\right]$

Hence we get,

$E\left(\hat{\alpha}_{B M L}{ }^{c}\right)=E\left[\left(\frac{K}{T}\right)^{c}\right]=K^{c} E\left[\left(T^{-c}\right)\right]$

And

$E\left(T^{-c}\right)=\int_{t} t^{-c} h(t) d t$

; $h(t)$ is function of gamm distribution

$=\frac{\alpha^{n}}{\Gamma(n)} \int_{0}^{\infty} e^{-\alpha t} t^{(n-c)-1} d t$

$=\frac{\alpha^{n} \Gamma(n-c)}{\Gamma(n) \alpha^{n-c}}=\frac{\alpha^{c} \Gamma(n-c)}{\Gamma(n)}$.

Using this result we get

$E\left(\hat{\alpha}_{B M L}{ }^{c}\right)=\frac{K^{c} \alpha^{c} \Gamma(n-c)}{\Gamma(n)}=\left\{\left(\frac{\Gamma(n)}{\Gamma(n-c)}\right)^{\frac{1}{c}}\right\}^{c} \frac{\alpha^{c} \Gamma(n-c)}{\Gamma(n)}=\alpha^{c}$

And 
$E\left(\ln \hat{\alpha}_{B M L}\right)=E\left[\ln \left(\frac{K}{T}\right)\right]=\ln K-E(\ln T)$

Here,

$E(\ln T)=\frac{\alpha^{n}}{\Gamma(n)} \int_{0}^{\infty} \ln t e^{-\alpha t} t^{n-1} d t$

Using the relation $\alpha t=y \Rightarrow t=\frac{1}{\alpha} y$

$\therefore d t=\frac{1}{\alpha} d y$

We get

$$
\begin{aligned}
& E(\ln T)=\frac{\alpha^{n}}{\Gamma(n)} \int_{0}^{\infty} \ln \left(\frac{1}{\alpha} y\right) e^{-y}\left(\frac{1}{\alpha} y\right)^{n-1} \frac{1}{\alpha} d y \\
& =\frac{-\ln \alpha}{\Gamma(n)} \int_{0}^{\infty} e^{-y} y^{n-1} d y+\frac{1}{\Gamma(n)} \int_{0}^{\infty} \ln y e^{-y} y^{n-1} d y \\
& =\frac{-\ln \alpha}{\Gamma(n)} \Gamma(n)+\frac{\Gamma^{\prime}(n)}{\Gamma(n)} \\
& =-\ln \alpha+\frac{\Gamma^{\prime}(n)}{\Gamma(n)}
\end{aligned}
$$

Where $\Gamma^{\prime}(n)=\int_{0}^{\infty} \ln y e^{-y} y^{n-1} d y$ is the first derivative of $\Gamma(n)$ with respect to $n$.

Using these results we get

$E\left(\ln \hat{\alpha}_{B M L}\right)=\ln K+\ln \alpha-\frac{\Gamma^{\prime}(n)}{\Gamma(n)}$.

Now the risk function becomes

$$
\begin{aligned}
& R_{M}(\alpha)=\omega\left[\frac{\alpha^{c}}{\alpha^{c}}+\operatorname{cln} \alpha-\operatorname{cln} \alpha-\operatorname{cln} K+\frac{c \Gamma^{\prime}(n)}{\Gamma(n)}-1\right] \\
& =\omega\left[\ln K^{-c}+\frac{c \Gamma^{\prime}(n)}{\Gamma(n)}\right]
\end{aligned}
$$

Which is constant w.r.t $\alpha$, as $\mathrm{c}$ and $\mathrm{n}$ are known and independent on.

So from Lehmann's theorem it follows that $d^{*}=\hat{\alpha}_{B}=\hat{\alpha}_{M M L}=$ $\left[\frac{\Gamma(n)}{\Gamma(n-c)}\right]^{\frac{1}{c}} \frac{1}{\sum_{i=1}^{n} \ln \left(1-e^{-\left(\lambda x_{i}\right)^{2}}\right)^{-1}}$ is the minimax estimator of the parameter $\alpha$ of the Generalized Rayleigh distribution under the MLINEX loss function (4)

As well-known, the maximum likelihood estimator (MLE) for the shape parameter of two parameter GR $(\alpha, \lambda)$ when $\lambda=$ 1 ( $\lambda$ is konwn), is

$$
\hat{\alpha}_{m l e}=-\frac{n}{\sum_{i=1}^{n} \ln \left(1-e^{-x_{i}^{2}}\right)}
$$

Note that, if $x_{i} \sim \operatorname{GR}(\alpha, 1)$, then $-\alpha \sum_{i=1}^{n} \ln \left(1-e^{-x_{i}^{2}}\right)$ follows Gamma distribution with shape parameter (n) and scale parameter 1; $\mathrm{G}(\mathrm{n}, 1)$.

i.e.; $E\left(\hat{\alpha}_{m l e}\right)=\frac{n}{n-1} \alpha$ and $\operatorname{var}\left(\hat{\alpha}_{m l e}\right)=\frac{n^{2}}{(n-1)^{2}(n-2)} \alpha^{2}$.

By using (7),

let $\hat{\alpha}_{B}=\frac{K}{n} \cdot \hat{\alpha}_{m l e}=\frac{K}{T} ; T=-\alpha \sum \ln \left(1-e^{-x^{2}}\right) \sim G(n, 1)$,

Then

$$
\begin{aligned}
& E\left(\hat{\alpha}_{B}\right)=\frac{K}{n} E\left(\hat{\alpha}_{m l e}\right)=\frac{K}{(n-1)} \alpha \\
& \therefore \operatorname{Bias}\left(\hat{\alpha}_{B}\right)=E\left(\hat{\alpha}_{B}\right)-\alpha=\frac{K \alpha-\alpha(n-1)}{n-1}
\end{aligned}
$$

$$
\begin{aligned}
& \operatorname{Var}\left(\hat{\alpha}_{B}\right)=\left(\frac{K}{n}\right)^{2} \cdot \operatorname{Var}\left(\hat{\alpha}_{m l e}\right)=\frac{K^{2}}{(n-1)^{2}(n-2)} \alpha^{2} \\
& \therefore \operatorname{MSE}\left(\hat{\alpha}_{B}\right)=\operatorname{Var}\left(\hat{\alpha}_{B}\right)+\left[\operatorname{Bias}\left(\hat{\alpha}_{B}\right)\right]^{2}=\frac{K^{2}}{(n-1)^{2}(n-2)} \alpha^{2}+\left(\frac{K \alpha-\alpha(n-1)}{n-1}\right)^{2} \\
& =\frac{K^{2}+K^{2}(n-2) \alpha^{2}-2 K(n-1)(n-2) \alpha^{2}+(n-2)(n-1)^{2} \alpha^{2}}{(n-1)^{2}(n-2)}
\end{aligned}
$$

\section{Double stage shrinkage estimator (DSSE) $\widetilde{\alpha}$}

In this section, we consider the (DSSE) $\tilde{\alpha}_{D S}$ which is defined in (3) using $\hat{\alpha}_{B}$ defined by $(8)$, when $\Psi\left(\hat{\alpha}_{1}\right)=h=\frac{k \alpha_{0}}{\widehat{\alpha} Z} ; Z=$ $X_{\left(1-\frac{\Delta}{2}, 2 n\right)}^{2} \& k=\left[\frac{\Gamma(n)}{\Gamma(n-c)}\right]^{\frac{1}{c}}$, such that $(0<\mathrm{h}<1)$ for estimate the shape parameter $\alpha$ of GR distribution when $\lambda=1$

$\tilde{\alpha}_{D S}=\left\{\begin{array}{l}h \hat{\alpha}_{B 1}+(1-h) \alpha_{0}, \text { if } \hat{\alpha}_{B 1} \in R \\ \hat{\alpha}_{p}=\frac{n 1 \widehat{\alpha}_{B 1}+n 2 \widehat{\alpha}_{B 2}}{n}, \text { if } \hat{\alpha}_{B 1} \notin R\end{array}\right.$

Where $\hat{\alpha}_{B 1}$ is the minimax estimator of $\alpha$ in the sample $(n i) ; i=$ 1,2. i. e. $\hat{\alpha}_{B 1}=\left[\frac{\Gamma(n i)}{\Gamma(n i-c)}\right]^{\frac{1}{c}} \frac{1}{\sum_{j=1}^{n i} \ln \left(1-e^{-\left(\lambda x_{j}\right)^{2}}\right)^{-1}}$, and $\mathrm{R}$ is a pretest region for testing the hypothesis $H_{0}: \alpha=\alpha_{0}$ vs. $H_{A}=\alpha \neq \alpha_{0}$ with level of significance $(\Delta)$ using test statistic function $T T=\frac{2 k \alpha_{0}}{\widehat{\alpha}_{B 1}}$

i.e. $; R=\left[a<\frac{2 k \alpha_{0}}{\widehat{\alpha}_{B 1}}<b\right]$

Where $a=X_{(1-\Delta, 2 n 1)}^{2}$ and $b=X^{2}{ }_{(\Delta, 2 n 1)}$

Are respectively the lower and upper $100(\Delta / 2)$ percentile point of chi-square distribution with degree of freedom $\left(2 \mathrm{n}_{1}\right)$.

The expression for Bias of DSSE $\left(\tilde{\alpha}_{D S}\right)$ is defined as below

$\operatorname{Bias}\left(\tilde{\alpha}_{D S} \mid \alpha ; R\right)=E\left(\tilde{\alpha}_{D S}-\alpha\right)=\int_{\widehat{\alpha}_{B 2}=0}^{\infty} \int_{\widehat{\alpha}_{B 1} \in R}\left[h\left(\hat{\alpha}_{B 1}-\alpha_{0}\right)+\right.$ $\left.\left(\alpha_{0}-\alpha\right)\right] f\left(\hat{\alpha}_{B 1}\right) f\left(\hat{\alpha}_{B 2}\right) d \hat{\alpha}_{B 1} d \hat{\alpha}_{B 2}+$

$\int_{\widehat{\alpha}_{B 2}=0}^{\infty} \int_{\hat{\alpha}_{B 1} \in \bar{R}}\left(\hat{\alpha}_{p}-\alpha\right) f\left(\hat{\alpha}_{B 1}\right) f\left(\hat{\alpha}_{B 2}\right) d \hat{\alpha}_{B 1} d \hat{\alpha}_{B 2}$

Where $\overline{\mathrm{R}}$ is the complement region of $\mathrm{R}$ in real space and $\hat{\alpha}_{B i} \sim I G(n, k \alpha)$ such that:

$f\left(\hat{\alpha}_{B i}\right)=\frac{\left(\frac{k \alpha}{\hat{\alpha}_{B i}}\right)^{n+1} \cdot e^{-\frac{k \alpha}{\hat{\alpha}_{B i}}}}{\Gamma(n) \cdot k \alpha} \hat{\alpha}_{B i}>0$

We conclude,

$\operatorname{Bias}\left(\tilde{\alpha}_{D S} \mid \alpha ; R\right)=\alpha\left\{\frac{k}{c} \zeta J_{0}\left(a^{*}, b^{*}\right)-\frac{1}{c} \zeta^{2} J^{\prime}{ }_{1}\left(a^{*}, b^{*}\right)+(\zeta-1) J_{0}\left(a^{*}, b^{*}\right)+\right.$ $\left(\frac{1}{1+u}\right)\left[\frac{k-(n 1-1)}{(n 1-1)}\right]+\left(\frac{u}{1+u}\right)\left[\frac{k-(n 2-1)}{(n 2-1)}\right]-\left(\frac{1}{1+u}\right)\left[k J_{1}\left(a^{*}, b^{*}\right)-J_{0}\left(a^{*}, b^{*}\right)\right]-$ $\left.\left(\frac{u}{1+u}\right)\left[\frac{k-(n 2-1)}{(n 2-1)}\right] J_{0}\left(a^{*}, b^{*}\right)\right\}$

Where $\mathrm{J}_{\ell}\left(\mathrm{a}^{*}, \mathrm{~b}^{*}\right)=\int_{\mathrm{a}^{*}}^{\mathrm{b}^{*}} \mathrm{y}^{-\ell} \frac{\mathrm{y}^{\mathrm{n}-1} \mathrm{e}^{-\mathrm{y}}}{\Gamma(\mathrm{n})} \mathrm{dy} ; \ell=0,1,2$

Also

$\zeta=\frac{\alpha_{0}}{\alpha}, a^{*}=(2 \zeta)^{-1} \cdot a, b^{*}=(2 \zeta)^{-1} \cdot b, u=\frac{n 2}{n 1}$ and $y=\left(k / \hat{\alpha}_{B 1}\right) \alpha$

The Bias ratio $[\mathrm{B}(\cdot)]$ of $\operatorname{DSSE}\left(\tilde{\alpha}_{D S}\right)$ is defined as:

$B\left(\tilde{\alpha}_{D S}\right)=\frac{\operatorname{Bias}\left(\widetilde{\alpha}_{D S} \mid \alpha ; R\right)}{\alpha}$

The expression of Mean squared error $[\operatorname{MSE}(\cdot)]$ of $\hat{\alpha}_{D S}$ derived as below:- 
$\operatorname{MSE}\left(\tilde{\alpha}_{D S} \mid \alpha ; R\right)=E\left(\tilde{\alpha}_{D S}-\alpha\right)^{2}=\int_{\widehat{\alpha}_{B 2}=0}^{\infty} \int_{\widehat{\alpha}_{B 1} \in R}\left[h\left(\hat{\alpha}_{B 1}-\alpha_{0}\right)+\right.$ $\left.\left(\alpha_{0}-\alpha\right)\right]^{2} f\left(\hat{\alpha}_{B 1}\right) f\left(\hat{\alpha}_{B 2}\right) d \hat{\alpha}_{B 1} d \hat{\alpha}_{B 2}+$

$\int_{\widehat{\alpha}_{B 2}=0}^{\infty} \int_{\widehat{\alpha}_{B 1} \in \bar{R}}\left(\hat{\alpha}_{p}-\alpha\right)^{2} f\left(\hat{\alpha}_{B 1}\right) f\left(\hat{\alpha}_{B 2}\right) d \hat{\alpha}_{B 1} d \hat{\alpha}_{B 2}$

And by simple computations, one can get:

$\operatorname{MSE}\left(\tilde{\alpha}_{D S} \mid \alpha ; R\right)=$

$\alpha^{2}\left\{\frac{k^{2}}{c^{2}} \zeta^{2} J_{0}\left(a^{*}, b^{*}\right)-2 \frac{k}{c^{2}} \zeta^{3} J^{\prime}{ }_{1}\left(a^{*}, b^{*}\right)+\frac{1}{c^{2}} \zeta^{4} J^{\prime}{ }_{2}\left(a^{*}, b^{*}\right)+2 \frac{k}{c} \zeta(\zeta-\right.$

1) $J_{0}\left(a^{*}, b^{*}\right)-2 \frac{1}{c} \zeta^{2}(\zeta-1) J^{\prime}{ }_{1}\left(a^{*}, b^{*}\right)+(\zeta-1)^{2} J_{0}\left(a^{*}, b^{*}\right)+$

$\left(\frac{1}{1+u}\right)^{2}\left[\frac{k^{2}+k^{2}(n 1-2)-2 k(n 1-1)(n 1-2)+(n 1-2)(n 1-1)^{2}}{(n 1-2)(n 1-1)^{2}}\right]+$

$2\left(\frac{u}{(1+u)^{2}}\right)\left[\frac{k-(n 1-1)}{(n 1-1)}\right]\left[\frac{k-(n 2-1)}{(n 2-1)}\right]+$

$\left(\frac{u}{1+u}\right)^{2}\left[\frac{k^{2}+k^{2}(n 2-2)-2 k(n 2-1)(n 2-2)+(n 2-2)(n 2-1)^{2}}{(n 2-2)(n 2-1)^{2}}\right]-\left(\frac{1}{1+u}\right)^{2}\left[k^{2} J_{1}\left(a^{*}, b^{*}\right)-\right.$

$\left.2 k J_{1}\left(a^{*}, b^{*}\right)+J_{0}\left(a^{*}, b^{*}\right)\right]-2\left(\frac{u}{(1+u)^{2}}\right)\left[\frac{k-(n 2-1)}{(n 2-1)}\right]\left[k J_{1}\left(a^{*}, b^{*}\right)-\right.$

$\left.\left.J_{0}\left(a^{*}, b^{*}\right)\right]-\left(\frac{u}{1+u}\right)^{2}\left[\frac{k^{2}+k^{2}(n 2-2)-2 k(n 2-1)(n 2-2)+(n 2-2)(n 2-1)^{2}}{(n 2-2)(n 2-1)^{2}}\right] J_{0}\left(a^{*}, b^{*}\right)\right\}$

Now, the Efficiency of $\tilde{\alpha}_{D S}$ relative to $\hat{\alpha}_{B 1}$ which is denoted by $R . E f f\left(\tilde{\alpha}_{D S} \mid \alpha ; R\right)$ is defined by:

$$
R . E f f\left(\tilde{\alpha}_{D S} \mid \alpha ; R\right)=\frac{M S E\left(\widehat{\alpha}_{B 1}\right)}{M S E\left(\widetilde{\alpha}_{D S} \mid \alpha ; R\right) \cdot[E(n / \alpha, R) / n]}
$$

Where $E(n / \alpha, R)$ is the Expected sample size, which is defined as:

$$
\mathrm{E}(\mathrm{n} / \alpha, \mathrm{R})=\mathrm{n}\left[1-\frac{\mathrm{u}}{1+\mathrm{u}} \mathrm{J}_{0}\left(\mathrm{a}^{*}, \mathrm{~b}^{*}\right)\right]
$$

As well as the Expected sample size proportion $E(n / \alpha, R) / n$ equal to

$$
1-\frac{\mathrm{u}}{1+\mathrm{u}} \mathrm{J}_{0}\left(\mathrm{a}^{*}, \mathrm{~b}^{*}\right)
$$

Also, we have to define the percentage of the overall sample saved (p.o.s.s.) of $\tilde{\alpha}_{D S}$ as:

$$
\text { p.o.s.s. }=\frac{\mathrm{n}_{2}}{\mathrm{n}} \mathrm{J}_{0}\left(\mathrm{a}^{*}, \mathrm{~b}^{*}\right) * 100
$$

And, finally, $p\left(\hat{\alpha}_{1} \in R\right)$ represent the probability of a voiding the second sample (stage).

See for example [1], [2]. [6], [9], and [17].

\section{Discussion and numerical results}

The computations of Relative Efficiency [R.Eff $(\cdot)$ ] and Bias Ratio [B (.)], Expected sample size $[\mathrm{E}(\mathrm{n} / \alpha, \mathrm{R})]$, Expected sample size proportion $[E(n / \alpha, R) / n]$, Percentage of the overall sample saved (p.o.s.s.) and probability of a voiding the second sample $\left[p\left(\hat{\alpha}_{1} \in\right.\right.$ $R)]$ were used for the estimator $\tilde{\alpha}_{D S}$. These computations were performed using Mathcad program for $\mathrm{n}_{1}=4,6,8,10,12, \mathrm{u}\left(=\mathrm{n}_{2} / \mathrm{n}_{1}\right)$
$=2,6,8,10,12, \zeta=\alpha_{0} / \alpha=0.25(0.25) 2, \Delta=0.01,0.05,0.1, \mathrm{c}=2$ and $\mathrm{h}$ $=\frac{k \alpha_{0}}{\widehat{\alpha} Z}$.

By using Mathcad program some of these computations are given in the tables (1)-(13).

The observation mentioned in the tables lead to the following results:

i) The Relative Efficiency [R.Eff( $\cdot)]$ of $\tilde{\alpha}_{D S}$ are adversely proportional with small value of $\Delta$ especially when $\zeta=1$, i.e. $\Delta$ $=0.01$ yield highest efficiency (see Tables (1), (5) and (9)).

ii) The Relative Efficiency [R.Eff (.)] of $\tilde{\alpha}_{D S}$ has maximum value when $\alpha=\alpha_{0}(\zeta=1)$, for each $\mathrm{n}_{1}, \Delta$ and decreasing otherwise $(\zeta \neq 1)$. This feature shown the important usefulness of prior knowledge which given higher effects of proposed estimator as well as the important role of shrinkage technique and its philosophy (see Tables (1), (5) and (9)).

iii) Bias ratio $[\mathrm{B}(\cdot)]$ of $\tilde{\alpha}_{D S}$ are reasonably small when $\alpha=\alpha_{0}$ for each $\mathrm{n}_{1}$, and same $\Delta$, and increases otherwise. This property shown that the proposed estimator $\widetilde{\alpha}_{\mathrm{DS}}$ is very closely to unbiasedness property especially when $\alpha=\alpha_{0}$ (see Tables (1), (5) and (9)).

iv) The Effective interval of $\widetilde{\alpha}_{D S}$ [the value of $\widetilde{\alpha}_{D S}$ which makes R.Eff( $(\cdot)$ of $\widetilde{\alpha}_{D S}$ greater than one] is approximate [0.25,2] (see Tables (1), (5) and (9)).

v) Bias ratio [B (.)] of $\widetilde{\alpha}_{\text {DS }}$ are reasonably large with small value of $u$ (see Tables (1), (5) and (9)).

vi) R.Eff $\left(\widetilde{\alpha}_{D S}\right)$ is decreasing function with increasing of the first sample size $\mathrm{n}_{1}$, for each $\Delta$ and $\zeta$ (see Tables (1), (5) and (9)).

vii) The Expected value of sample size of $\widetilde{\alpha}_{D S}$ is close to $n_{1}$, especially when $\zeta \cong 1$ and start faraway otherwise (see Tables (2), (6) and (10)).

viii) Percentage of the overall sample saved $\left[\frac{\mathrm{n}_{2}}{\mathrm{n}} \mathrm{J}_{0}\left(\mathrm{a}^{*}, \mathrm{~b}^{*}\right) * 100\right]$ is increasing value with increasing value of $\mathrm{u}\left(\mathrm{u}=\mathrm{n}_{2} / \mathrm{n}_{1}\right)$ and $\zeta$ (see Tables (4), (8) and (12)).

ix) R.Eff $\left(\widetilde{\alpha}_{D S}\right)$ is an increasing function with respect to u. This property shown the effective of proposed estimator using small $\mathrm{n}_{1}$ relative to $\mathrm{n}_{2}$ (or large $\mathrm{n}_{2}$ ) which given higher efficiency and reduce the observation cost (see Tables (1), (5) and (9)).

$\mathrm{x}$ ) The considered estimator $\widetilde{\alpha}_{\mathrm{DS}}$ is better than the minimax estimator especially when $\alpha \approx \alpha_{0}$, this will give the effective of $\widetilde{\alpha}_{D S}$ relative to $\widehat{\alpha}_{B 1}$ and also given an important weight of prior knowledge, and the augmentation of efficiency may be reach to ten times (see Tables (1), (5) and (9)).

xi) The probability of avoiding second sample of the considered estimator $\tilde{\alpha}_{\mathrm{DS}}$ has a maximum value when $\alpha=\alpha_{0}$ for each $\mathrm{n}_{1}$ and $\Delta$ (see Tables (13)), this property shown the effective of double stage shrinkage estimators to reduce the sample size.

xii) The considered estimator $\tilde{\alpha}_{\mathrm{DS}}$ is more efficient than the esti-

\begin{tabular}{|c|c|c|c|c|c|c|c|c|c|c|}
\hline \multirow[b]{2}{*}{$\Delta$} & \multirow[b]{2}{*}{$\mathrm{n}_{1}$} & \multirow[b]{2}{*}{$\begin{array}{l}\text { R.Eff(-) } \\
\text { B(-) }\end{array}$} & \multicolumn{8}{|c|}{$\zeta$} \\
\hline & & & 0.25 & 0.50 & 0.75 & 1 & 1.25 & 1.50 & 1.75 & 2 \\
\hline \multirow{3}{*}{$\stackrel{\circ}{\circ}$} & 4 & $\begin{array}{l}\text { R.Eff(-) } \\
\text { B(-) }\end{array}$ & $\begin{array}{l}3.8375436 \\
-0.7361405\end{array}$ & $\begin{array}{l}17.0374954 \\
-0.501523\end{array}$ & $\begin{array}{l}70.8568096 \\
-0.501523\end{array}$ & $\begin{array}{l}349.5356128 \\
-6.033328 E-3\end{array}$ & $\begin{array}{l}54.9780952 \\
0.2202785\end{array}$ & $\begin{array}{l}14.0415248 \\
0.4065392\end{array}$ & $\begin{array}{l}5.4920024 \\
0.5400779\end{array}$ & $\begin{array}{l}2.7364843 \\
0.6198681\end{array}$ \\
\hline & 6 & $\begin{array}{l}\text { R.Eff(-) } \\
\text { B(-) }\end{array}$ & $\begin{array}{l}2.2357931 \\
-0.7372543\end{array}$ & $\begin{array}{l}13.2897009 \\
-0.5103292\end{array}$ & $\begin{array}{l}67.9836612 \\
-0.2546055\end{array}$ & $\begin{array}{l}446.9918537 \\
-6.621779 E-3\end{array}$ & $\begin{array}{l}52.0188327 \\
0.2142392\end{array}$ & $\begin{array}{l}12.0565522 \\
0.3754864\end{array}$ & $\begin{array}{l}4.4887373 \\
0.45856\end{array}$ & $\begin{array}{l}2.2291462 \\
0.4679973\end{array}$ \\
\hline & 10 & $\begin{array}{l}\text { R.Eff(-) } \\
\text { B(-) }\end{array}$ & $\begin{array}{l}1.481969 \\
-0.7380109\end{array}$ & $\begin{array}{l}7.8517441 \\
-0.5368034\end{array}$ & $\begin{array}{l}60.3960552 \\
-0.2600615\end{array}$ & $\begin{array}{l}568.5749596 \\
-6.959501 \mathrm{E}-3\end{array}$ & $\begin{array}{l}46.1709369 \\
0.2023992\end{array}$ & $\begin{array}{l}9.3115594 \\
0.3100801\end{array}$ & $\begin{array}{l}3.3327567 \\
0.293013\end{array}$ & $\begin{array}{l}1.747586 \\
0.1833459\end{array}$ \\
\hline
\end{tabular}
mators introduced by [7], [9] and [11] in the sense of higher efficiency.

Table 1: Shown Bias Ratio $[\mathrm{B}(\cdot)]$ and R.E.Ff of $\widetilde{\mathrm{A}}_{\mathrm{DS}}$ W.R.T. $\Delta, \mathrm{N}_{1}$ and $\zeta$ when $\mathrm{U}=6$ 


\begin{tabular}{|c|c|c|c|c|c|c|c|c|c|c|}
\hline & 12 & $\begin{array}{l}\text { R.Eff(-) } \\
\text { B(-) }\end{array}$ & $\begin{array}{l}1.4170837 \\
-0.7358799\end{array}$ & $\begin{array}{l}6.1274647 \\
-0.5532719\end{array}$ & $\begin{array}{c}56.4217269 \\
-0.2630556\end{array}$ & $\begin{array}{l}609.5683911 \\
-7.021246 \mathrm{E}-3\end{array}$ & $\begin{array}{l}43.461959 \\
0.1963193\end{array}$ & $\begin{array}{l}8.2932353 \\
0.2760166\end{array}$ & $\begin{array}{l}2.9697037 \\
0.2117398\end{array}$ & $\begin{array}{l}1.6255451 \\
0.0565818\end{array}$ \\
\hline \multirow{3}{*}{ 号 } & 4 & $\begin{array}{l}\text { R.Eff(-) } \\
\text { B(-) }\end{array}$ & $\begin{array}{l}2.0367028 \\
-0.7560093\end{array}$ & $\begin{array}{l}9.4204389 \\
-0.5288025\end{array}$ & $\begin{array}{c}45.3879879 \\
-0.2706274\end{array}$ & $\begin{array}{l}121.737409 \\
-0.0339792\end{array}$ & $\begin{array}{l}28.4121193 \\
0.1509111\end{array}$ & $\begin{array}{l}8.3573908 \\
0.2681358\end{array}$ & $\begin{array}{l}3.6165638 \\
0.321622\end{array}$ & $\begin{array}{l}2.0052434 \\
0.3255413\end{array}$ \\
\hline & 6 & $\begin{array}{l}\text { R.Eff(-) } \\
\text { B(-) }\end{array}$ & $\begin{array}{l}1.531913 \\
-0.7526109\end{array}$ & $\begin{array}{l}6.4309968 \\
-0.5520324\end{array}$ & $\begin{array}{l}39.4013805 \\
-0.2786634\end{array}$ & $\begin{array}{l}132.4978401 \\
-0.0346019\end{array}$ & $\begin{array}{l}25.7079751 \\
0.1376973\end{array}$ & $\begin{array}{l}6.9676297 \\
0.2142247\end{array}$ & $\begin{array}{l}2.9899699 \\
0.2063821\end{array}$ & $\begin{array}{l}1.7201642 \\
0.1428628\end{array}$ \\
\hline & 10 & $\begin{array}{l}\text { R.Eff(-) } \\
\text { B(-) }\end{array}$ & $\begin{array}{l}1.3830997 \\
-0.7414922\end{array}$ & $\begin{array}{l}3.6073693 \\
-0.6009981\end{array}$ & $\begin{array}{l}29.5192187 \\
-0.2952873\end{array}$ & $\begin{array}{c}141.123789 \\
-0.0351368\end{array}$ & $\begin{array}{l}21.3533649 \\
0.1121118\end{array}$ & $\begin{array}{l}5.2537323 \\
0.1112742\end{array}$ & $\begin{array}{l}2.3339035 \\
2.8841824 \mathrm{E}-3\end{array}$ & $\begin{array}{l}1.4890989 \\
-0.1467708\end{array}$ \\
\hline \multirow{5}{*}{$\stackrel{0}{-}$} & 12 & $\begin{array}{l}\text { R.Eff(-) } \\
\text { B(-) }\end{array}$ & $\begin{array}{l}1.3850913 \\
-0.7370681\end{array}$ & $\begin{array}{l}2.9271336 \\
-0.623249\end{array}$ & $\begin{array}{l}25.7390545 \\
-0.3038255\end{array}$ & $\begin{array}{l}143.3368334 \\
-0.0352589\end{array}$ & $\begin{array}{l}19.5935066 \\
0.0994719\end{array}$ & $\begin{array}{l}4.6787938 \\
0.0623025\end{array}$ & $\begin{array}{l}2.1416783 \\
-0.0853298\end{array}$ & $\begin{array}{l}1.4416449 \\
-0.2577666\end{array}$ \\
\hline & 6 & $\begin{array}{l}\text { R.Eff(-) } \\
\text { B(-) }\end{array}$ & $\begin{array}{l}1.4051012 \\
-0.7581989\end{array}$ & $\begin{array}{c}4.2510831 \\
-0.588899\end{array}$ & $\begin{array}{l}23.8450315 \\
-0.3085423\end{array}$ & $\begin{array}{c}58.2022606 \\
-0.0710792\end{array}$ & $\begin{array}{l}15.7526535 \\
0.0654675\end{array}$ & $\begin{array}{l}5.0976112 \\
0.0957881\end{array}$ & $\begin{array}{l}2.4700569 \\
0.0509138\end{array}$ & $\begin{array}{l}1.5607989 \\
-0.0331327\end{array}$ \\
\hline & 8 & $\begin{array}{l}\text { R.Eff(-) } \\
\text { B(-) }\end{array}$ & $\begin{array}{l}1.369939 \\
-0.7487252\end{array}$ & $\begin{array}{l}3.1978839 \\
-0.6165186\end{array}$ & $\begin{array}{l}20.119457 \\
-0.3204947\end{array}$ & $\begin{array}{c}59.4843514 \\
-0.0711455\end{array}$ & $\begin{array}{l}14.3148601 \\
0.0491657\end{array}$ & $\begin{array}{l}4.4292617 \\
0.0368678\end{array}$ & $\begin{array}{l}2.1982133 \\
-0.0570746\end{array}$ & $\begin{array}{l}1.4683349 \\
-0.1798678\end{array}$ \\
\hline & 10 & $\begin{array}{l}\text { R.Eff(-) } \\
\text { B(-) }\end{array}$ & $\begin{array}{l}1.3741103 \\
-0.7419052\end{array}$ & $\begin{array}{l}2.5822991 \\
-0.6405443\end{array}$ & $\begin{array}{c}17.1643208 \\
-0.3325064\end{array}$ & $\begin{array}{l}60.2163193 \\
-0.0712066\end{array}$ & $\begin{array}{l}13.0867277 \\
0.0331166\end{array}$ & $\begin{array}{l}3.9307041 \\
-0.0186425\end{array}$ & $\begin{array}{l}2.0143657 \\
-0.1517676\end{array}$ & $\begin{array}{l}1.421593 \\
-0.2977595\end{array}$ \\
\hline & 12 & $\begin{array}{l}\text { R.Eff(-) } \\
\text { B(-) }\end{array}$ & $\begin{array}{l}1.3831841 \\
-0.7371543\end{array}$ & $\begin{array}{l}2.2003462 \\
-0.6604705\end{array}$ & $\begin{array}{l}14.8128696 \\
-0.3444782\end{array}$ & $\begin{array}{l}60.6927863 \\
-0.071251\end{array}$ & $\begin{array}{l}12.0277307 \\
0.0173067\end{array}$ & $\begin{array}{l}3.5442343 \\
-0.0708363\end{array}$ & $\begin{array}{l}1.8831179 \\
-0.2343807\end{array}$ & $\begin{array}{l}1.3987802 \\
-0.3917032\end{array}$ \\
\hline
\end{tabular}

Table 2: Shown Expected Sample Size of $\tilde{\alpha}$ W.R.T. $\Delta$, U, and $\zeta U=6$

\begin{tabular}{|c|c|c|c|c|c|c|c|c|c|c|}
\hline \multirow{3}{*}{$\mathrm{u}$} & \multirow[b]{3}{*}{$\mathrm{n}_{1}$} & \multirow[b]{3}{*}{$\Delta$} & \multirow{2}{*}{\multicolumn{8}{|c|}{$\zeta$}} \\
\hline & & & & & & & & & & \\
\hline & & & 0.25 & 0.50 & 0.75 & 1 & 1.50 & 1.25 & 1.75 & 2 \\
\hline \multirow{14}{*}{6} & \multirow{3}{*}{4} & 0.01 & 10.798 & 5.143 & 4.325 & 4.240 & 4.648 & 5.627 & 7.099 & 8.881 \\
\hline & & 0.05 & 19.205 & 8.242 & 5.508 & 5.200 & 6.236 & 8.131 & 10.419 & 12.752 \\
\hline & & 0.1 & 23.066 & 11.040 & 6.888 & 6.401 & 7.822 & 10.147 & 12.700 & 15.117 \\
\hline & \multirow{3}{*}{6} & 0.01 & 26.800 & 9.296 & 6.669 & 6.360 & 7.175 & 9.329 & 12.617 & 16.490 \\
\hline & & 0.05 & 37.397 & 16.108 & 8.862 & 7.800 & 9.824 & 13.789 & 18.493 & 23.051 \\
\hline & & 0.1 & 40.135 & 21.252 & 11.247 & 9.600 & 12.355 & 17.108 & 22.158 & 26.640 \\
\hline & \multirow{2}{*}{8} & 0.01 & 46.605 & 15.134 & 9.160 & 8.480 & 9.844 & 13.681 & 19.512 & 26.099 \\
\hline & & 0.1 & 55.498 & 34.003 & 16.225 & 12.801 & 17.289 & 25.188 & 33.207 & 39.823 \\
\hline & \multirow{3}{*}{10} & 0.01 & 65.545 & 22.965 & 11.820 & 10.600 & 12.661 & 18.713 & 27.761 & 37.483 \\
\hline & & 0.05 & 69.521 & 39.398 & 16.878 & 13.000 & 17.935 & 28.089 & 39.309 & 48.859 \\
\hline & & 0.1 & 69.892 & 48.586 & 21.812 & 16.001 & 22.616 & 34.290 & 45.532 & 54.116 \\
\hline & \multirow{3}{*}{12} & 0.01 & 82.277 & 32.904 & 14.665 & 12.720 & 15.634 & 24.447 & 37.308 & 50.366 \\
\hline & & 0.05 & 83.886 & 54.006 & 21.566 & 15.600 & 22.458 & 36.636 & 51.611 & 63.466 \\
\hline & & 0.1 & 83.980 & 64.339 & 28.000 & 19.199 & 28.327 & 44.315 & 58.859 & 69.103 \\
\hline
\end{tabular}

Table 3: Shown Expected Sample Size Proportion W.R.T. $\Delta, \mathrm{U}, \mathrm{N}_{1}$ and $\zeta$

\begin{tabular}{|c|c|c|c|c|c|c|c|c|c|c|}
\hline \multirow{3}{*}{$\mathrm{u}$} & \multirow[b]{3}{*}{$\mathrm{n}_{1}$} & \multirow[b]{3}{*}{$\Delta$} & \multirow{2}{*}{\multicolumn{8}{|c|}{$\zeta$}} \\
\hline & & & & & & & & & & \\
\hline & & & 0.25 & 0.50 & 0.75 & 1 & 1.25 & 1.50 & 1.75 & 2 \\
\hline \multirow{15}{*}{6} & \multirow{3}{*}{4} & 0.01 & 0.386 & 0.184 & 0.154 & 0.151424 & 0.166 & 0.201 & 0.254 & 0.317 \\
\hline & & 0.05 & 0.686 & 0.294 & 0.197 & 0.185719 & 0.223 & 0.290 & 0.372 & 0.455 \\
\hline & & 0.1 & 0.824 & 0.394 & 0.246 & 0.22859 & 0.279 & 0.362 & 0.454 & 0.540 \\
\hline & \multirow{3}{*}{6} & 0.01 & 0.638 & 0.221 & 0.159 & 0.15142 & 0.171 & 0.222 & 0.300 & 0.393 \\
\hline & & 0.05 & 0.890 & 0.384 & 0.211 & 0.185716 & 0.234 & 0.328 & 0.440 & 0.549 \\
\hline & & 0.1 & 0.956 & 0.506 & 0.268 & 0.22857 & 0.294 & 0.407 & 0.528 & 0.634 \\
\hline & \multirow{3}{*}{8} & 0.01 & 0.832 & 0.270 & 0.164 & 0.15143 & 0.176 & 0.244 & 0.348 & 0.466 \\
\hline & & 0.05 & 0.970 & 0.475 & 0.226 & 0.1857216 & 0.245 & 0.365 & 0.504 & 0.629 \\
\hline & & 0.1 & 0.991 & 0.607 & 0.290 & 0.22858 & 0.309 & 0.45 & 0.593 & 0.711 \\
\hline & \multirow{3}{*}{10} & 0.01 & 0.936 & 0.328 & 0.169 & 0.151 & 0.181 & 0.267 & 0.397 & 0.535 \\
\hline & & 0.05 & 0.993 & 0.563 & 0.241 & 0.1857 & 0.256 & 0.401 & 0.562 & 0.698 \\
\hline & & 0.1 & 0.998 & 0.694 & 0.312 & 0.22857 & 0.323 & 0.490 & 0.65 & 0.773 \\
\hline & \multirow{3}{*}{12} & 0.01 & 0.979 & 0.392 & 0.175 & 0.151 & 0.186 & 0.291 & 0.444 & 0.600 \\
\hline & & 0.05 & 0.999 & 0.643 & 0.257 & 0.186 & 0.267 & 0.436 & 0.614 & 0.756 \\
\hline & & 0.1 & 1 & 0.766 & 0.333 & 0.229 & 0.337 & 0.528 & 0.701 & 0.823 \\
\hline
\end{tabular}

Table 4: Shown Percentage of Overall Sample Saved W.R.T. $\Delta, U, N_{1}$ and $\zeta$

\begin{tabular}{|c|c|c|c|c|c|c|c|c|c|c|}
\hline \multirow[b]{2}{*}{$\mathrm{u}$} & \multirow[b]{2}{*}{$\mathrm{n}_{1}$} & \multirow[b]{2}{*}{$\Delta$} & \multicolumn{8}{|c|}{$\zeta$} \\
\hline & & & 0.25 & 0.50 & 0.75 & 1 & 1.25 & 1.50 & 1.75 & 2 \\
\hline \multirow{3}{*}{6} & & 0.01 & 61.435 & 81.633 & 84.553 & 84.858 & 83.399 & 79.904 & 74.646 & 68.281 \\
\hline & 4 & 0.05 & 31.411 & 70.564 & 80.329 & 81.4281 & 77.729 & 70.961 & 62.790 & 54.456 \\
\hline & & 0.1 & 17.621 & 60.571 & 75.400 & 77.140 & 72.065 & 63.762 & 54.642 & 46.011 \\
\hline
\end{tabular}




\begin{tabular}{|c|c|c|c|c|c|c|c|c|c|}
\hline & 0.01 & 36.189 & 77.866 & 84.122 & 84.8571 & 82.917 & 77.788 & 69.959 & 60.739 \\
\hline \multirow[t]{3}{*}{6} & 0.05 & 10.959 & 61.648 & 78.900 & 81.4284 & 76.609 & 67.170 & 55.968 & 45.118 \\
\hline & 0.1 & 4.440 & 49.400 & 73.222 & 77.143 & 70.584 & 59.267 & 47.242 & 36.571 \\
\hline & 0.01 & 16.776 & 72.976 & 83.642 & 84.85722 & 82.422 & 75.569 & 65.158 & 53.394 \\
\hline \multirow[t]{3}{*}{8} & 0.05 & 2.998 & 52.493 & 77.416 & 81.427 & 75.491 & 63.469 & 49.645 & 37.054 \\
\hline & 0.1 & 0.897 & 39.28 & 71.027 & 77.141 & 69.128 & 55.021 & 40.703 & 28.888 \\
\hline & 0.01 & 6.364 & 67.192 & 83.114 & 84.857 & 81.912 & 73.267 & 60.341 & 46.453 \\
\hline \multirow[t]{2}{*}{10} & 0.05 & 0.685 & 43.716 & 75.889 & 81.428 & 74.378 & 59.874 & 43.844 & 30.202 \\
\hline & 0.1 & 0.155 & 30.591 & 68.84 & 77.142 & 67.692 & 51.015 & 34.954 & 22.691 \\
\hline \multirow[t]{2}{*}{12} & 0.05 & 0.136 & 35.707 & 74.326 & 81.429 & 73.264 & 56.385 & 38.559 & 24.445 \\
\hline & 0.1 & 0.024 & 23.406 & 66.667 & 77.144 & 66.278 & 47.245 & 29.929 & 17.735 \\
\hline
\end{tabular}

Table 5: Shown Bias Ratio $[\mathrm{B}(\cdot)]$ and R.E.Ff of $\widetilde{\mathrm{A}}_{\mathrm{DS}}$ W.R.T. $\Delta, \mathrm{N}_{1}$ and $\zeta$ when $\mathrm{U}=10$

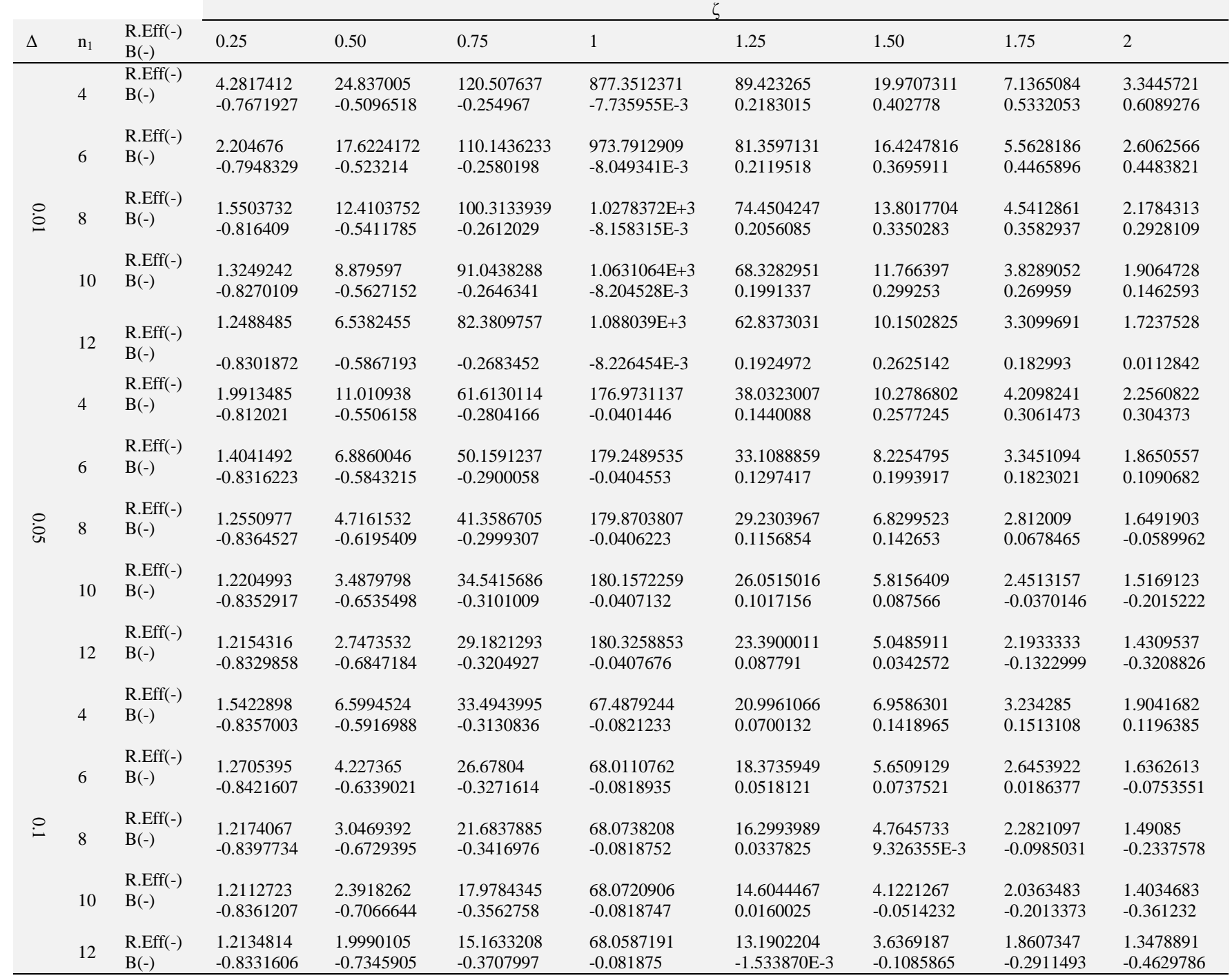

Table 6: Shown Expected Sample Size of $\tilde{\alpha}$ W.R.T. $\Delta$, U, and $\zeta$

\begin{tabular}{|c|c|c|c|c|c|c|c|c|c|c|}
\hline \multirow[b]{2}{*}{$\mathrm{u}$} & \multirow[b]{2}{*}{$\mathrm{n}_{1}$} & \multirow[b]{2}{*}{$\Delta$} & \multicolumn{8}{|c|}{$\zeta$} \\
\hline & & & 0.25 & 0.50 & 0.75 & 1 & 1.50 & 1.25 & 1.75 & 2 \\
\hline & & 0.01 & 15.331 & 5.905 & 4.542 & 4.400 & 5.081 & 6.711 & 9.165 & 12.135 \\
\hline & 4 & 0.05 & 29.341 & 11.070 & 6.513 & 6.000 & 7.726 & 10.885 & 14.698 & 18.587 \\
\hline & & 0.1 & 35.777 & 15.733 & 8.813 & 8.001 & 10.370 & 14.244 & 18.500 & 22.528 \\
\hline & & 0.01 & 40.667 & 11.494 & 7.115 & 6.600 & 7.958 & 11.548 & 17.029 & 23.483 \\
\hline & 6 & 0.05 & 58.329 & 22.846 & 10.770 & 9.000 & 12.374 & 18.981 & 26.822 & 34.418 \\
\hline & & 0.1 & 62.892 & 31.420 & 14.745 & 12.000 & 16.591 & 24.513 & 32.931 & 40.400 \\
\hline & & 0.01 & 72.342 & 19.889 & 9.934 & 8.800 & 11.073 & 17.469 & 27.186 & 38.166 \\
\hline & & 0.1 & 87.163 & 51.338 & 21.708 & 16.001 & 23.481 & 36.647 & 50.011 & 61.038 \\
\hline & & 0.01 & 102.575 & 31.609 & 13.033 & 11.000 & 14.436 & 24.522 & 39.602 & 55.805 \\
\hline & 10 & 0.05 & 109.201 & 58.997 & 21.463 & 15.000 & 23.225 & 40.148 & 58.849 & 74.765 \\
\hline & & 0.1 & 109.819 & 74.311 & 29.687 & 20.001 & 31.026 & 50.483 & 69.220 & 83.527 \\
\hline & & 0.01 & 129.129 & 46.840 & 16.442 & 13.200 & 18.056 & 32.745 & 54.180 & 75.943 \\
\hline & 12 & 0.05 & 131.809 & 82.010 & 27.943 & 18.000 & 29.430 & 53.061 & 78.018 & 97.777 \\
\hline 은 & & 0.1 & 131.967 & 99.232 & 38.666 & 23.999 & 39.211 & 65.858 & 90.099 & 107.171 \\
\hline
\end{tabular}


Table 7: Shown Expected Sample Size Proportion W.R.T. $\Delta, \mathrm{U}, \mathrm{N}_{1}$ and $\zeta$

\begin{tabular}{|c|c|c|c|c|c|c|c|c|c|c|}
\hline \multirow[b]{2}{*}{$\mathrm{u}$} & \multirow[b]{2}{*}{$\mathrm{n}_{1}$} & \multirow[b]{2}{*}{$\Delta$} & \\
\hline & & & 0.25 & 0.50 & 0.75 & 1 & 1.25 & 1.50 & 1.75 & 2 \\
\hline \multirow{14}{*}{10} & \multirow{3}{*}{4} & 0.01 & 0.348 & 0.134 & 0.103 & 0.099995 & 0.115 & 0.153 & 0.208 & 0.276 \\
\hline & & 0.05 & 0.667 & 0.252 & 0.148 & 0.136368 & 0.176 & 0.247 & 0.334 & 0.422 \\
\hline & & 0.1 & 0.813 & 0.358 & 0.200 & 0.18184 & 0.236 & 0.324 & 0.420 & 0.512 \\
\hline & \multirow[t]{2}{*}{6} & 0.05 & 0.884 & 0.346 & 0.163 & 0.136365 & 0.187 & 0.288 & 0.406 & 0.521 \\
\hline & & 0.1 & 0.953 & 0.476 & 0.223 & 0.18182 & 0.251 & 0.371 & 0.499 & 0.612 \\
\hline & \multirow{3}{*}{8} & 0.01 & 0.822 & 0.226 & 0.113 & 0.0999992 & 0.126 & 0.199 & 0.309 & 0.434 \\
\hline & & 0.05 & 0.968 & 0.443 & 0.179 & 0.13637 & 0.199 & 0.327 & 0.473 & 0.607 \\
\hline & & 0.1 & 0.99 & 0.583 & 0.247 & 0.18183 & 0.267 & 0.416 & 0.568 & 0.694 \\
\hline & \multirow{3}{*}{10} & 0.01 & 0.933 & 0.287 & 0.118 & 0.1000004 & 0.131 & 0.223 & 0.36 & 0.507 \\
\hline & & 0.05 & 0.993 & 0.536 & 0.195 & 0.136364 & 0.211 & 0.365 & 0.535 & 0.68 \\
\hline & & 0.1 & 0.998 & 0.676 & 0.27 & 0.181827 & 0.282 & 0.459 & 0.629 & 0.759 \\
\hline & \multirow{3}{*}{12} & 0.01 & 0.978 & 0.355 & 0.125 & 0.099998 & 0.137 & 0.248 & 0.41 & 0.575 \\
\hline & & 0.05 & 0.999 & 0.621 & 0.212 & 0.136362 & 0.223 & 0.402 & 0.591 & 0.741 \\
\hline & & 0.1 & 1 & 0.752 & 0.293 & 0.18181 & 0.297 & 0.499 & 0.683 & 0.812 \\
\hline
\end{tabular}

Table 8: Shown Percentage of Overall Sample Saved W.R.T. $\Delta, U, N_{1}$ and $\zeta$

\begin{tabular}{|c|c|c|c|c|c|c|c|c|c|c|}
\hline \multirow[b]{2}{*}{$\mathrm{u}$} & \multirow[b]{2}{*}{$\mathrm{n}_{1}$} & \multirow[b]{2}{*}{$\Delta$} & \multicolumn{8}{|c|}{$\zeta$} \\
\hline & & & 0.25 & 0.50 & 0.75 & 1 & 1.25 & 1.50 & 1.75 & 2 \\
\hline \multirow{15}{*}{10} & \multirow{3}{*}{4} & 0.01 & 65.158 & 86.581 & 89.678 & 90.0004 & 88.453 & 84.747 & 79.170 & 72.420 \\
\hline & & 0.05 & 33.315 & 74.840 & 85.197 & 86.3631 & 82.44 & 75.261 & 66.595 & 57.757 \\
\hline & & 0.1 & 18.689 & 64.242 & 79.97 & 81.815 & 76.432 & 67.626 & 57.954 & 48.800 \\
\hline & \multirow{3}{*}{6} & 0.01 & 38.383 & 82.585 & 89.22 & 89.99995 & 87.942 & 82.503 & 74.199 & 64.42 \\
\hline & & 0.05 & 11.623 & 65.385 & 83.682 & 86.3634 & 81.252 & 71.241 & 59.36 & 47.852 \\
\hline & & 0.1 & 4.709 & 52.394 & 77.659 & 81.8181 & 74.862 & 62.859 & 50.105 & 38.788 \\
\hline & \multirow{3}{*}{8} & 0.01 & 17.793 & 77.398 & 88.712 & 90.0000 & 87.417 & 80.149 & 69.107 & 56.63 \\
\hline & & 0.05 & 3.179 & 55.674 & 82.108 & 86.3629 & 80.067 & 67.316 & 52.654 & 39.3 \\
\hline & & 0.1 & 0.951 & 41.661 & 75.332 & 81.816 & 73.317 & 58.356 & 43.169 & 30.639 \\
\hline & \multirow{3}{*}{10} & 0.01 & 6.75 & 71.265 & 88.152 & 89.99996 & 86.877 & 77.707 & 63.998 & 49.268 \\
\hline & & 0.05 & 0.726 & 46.366 & 80.488 & 86.3636 & 78.886 & 63.502 & 46.501 & 32.032 \\
\hline & & 0.1 & 0.164 & 32.445 & 73.012 & 81.8172 & 71.795 & 54.107 & 37.073 & 24.067 \\
\hline & \multirow{3}{*}{12} & 0.01 & 2.175 & 64.515 & 87.544 & 90.0001 & 86.321 & 75.193 & 58.955 & 42.468 \\
\hline & & 0.05 & 0.145 & 37.871 & 78.831 & 86.36378 & 77.704 & 59.803 & 40.896 & 25.927 \\
\hline & & 0.1 & 0.025 & 24.825 & 70.708 & 81.8189 & 70.294 & 50.108 & 31.743 & 18.81 \\
\hline
\end{tabular}

Table 9: Shown Bias Ratio [B (.)] and R.E.Ff of $\widetilde{\mathrm{A}}_{\mathrm{DS}}$ W.R.T. $\Delta, \mathrm{N}_{1}$ and $\zeta$ when $\mathrm{U}=12$

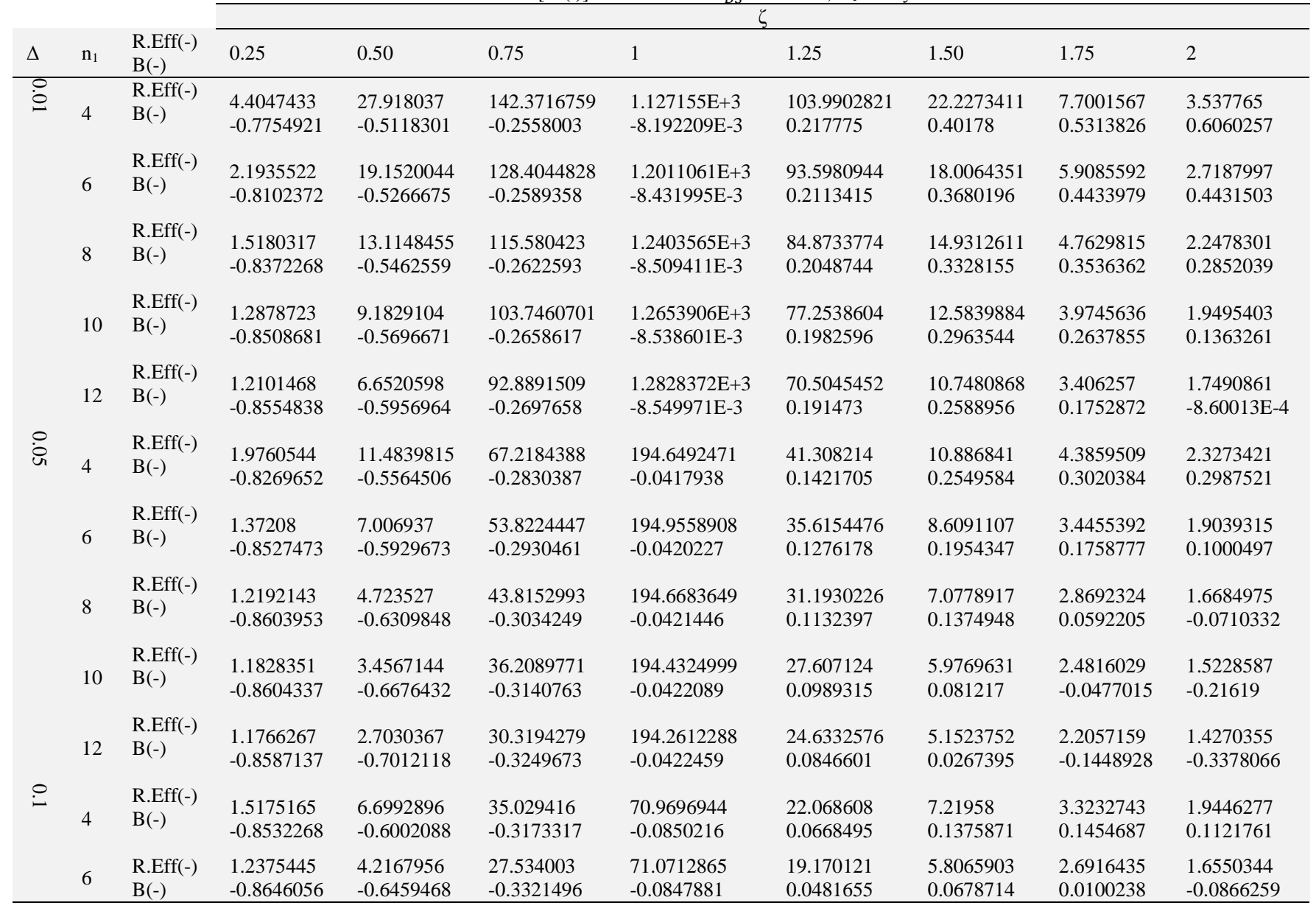




\begin{tabular}{|c|c|c|c|c|c|c|c|c|c|}
\hline 8 & $\begin{array}{l}\text { R.Eff(-) } \\
\text { B(-) }\end{array}$ & $\begin{array}{l}1.1814512 \\
-0.8641521\end{array}$ & $\begin{array}{l}3.0076239 \\
-0.6880563\end{array}$ & $\begin{array}{l}22.1709488 \\
-0.3473829\end{array}$ & $\begin{array}{l}70.9534873 \\
-0.0847505\end{array}$ & $\begin{array}{l}16.9039944 \\
0.0296672\end{array}$ & $\begin{array}{l}4.8565672 \\
1.95993 \mathrm{E}-3\end{array}$ & $\begin{array}{l}2.302899 \\
-0.1095857\end{array}$ & $\begin{array}{l}1.4953222 \\
-0.2481767\end{array}$ \\
\hline 10 & $\begin{array}{l}\text { R.Eff(-) } \\
\text { B(-) }\end{array}$ & $\begin{array}{l}1.1736085 \\
-0.8613739\end{array}$ & $\begin{array}{l}2.3451492 \\
-0.7243935\end{array}$ & $\begin{array}{l}18.2477612 \\
-0.362653\end{array}$ & $\begin{array}{l}70.8512733 \\
-0.0847356\end{array}$ & $\begin{array}{l}15.067225 \\
0.0114186\end{array}$ & $\begin{array}{l}4.1735307 \\
-0.0602031\end{array}$ & $\begin{array}{l}2.0407626 \\
-0.2146162\end{array}$ & $\begin{array}{l}1.3976371 \\
-0.3782379\end{array}$ \\
\hline 12 & $\begin{array}{l}\text { R.Eff(-) } \\
\text { B(-) }\end{array}$ & $\begin{array}{l}1.1746785 \\
-0.8589123 \\
\end{array}$ & $\begin{array}{l}1.9512073 \\
-0.754476 \\
\end{array}$ & $\begin{array}{c}15.2986375 \\
-0.3778649 \\
\end{array}$ & $\begin{array}{r}70.7737677 \\
-0.0847257 \\
\end{array}$ & $\begin{array}{l}13.5454418 \\
-6.58452 \mathrm{E}-3 \\
\end{array}$ & $\begin{array}{l}3.6614247 \\
-0.1187069 \\
\end{array}$ & $\begin{array}{l}1.8539925 \\
-0.3063706 \\
\end{array}$ & $\begin{array}{l}1.3342339 \\
-0.4820918 \\
\end{array}$ \\
\hline
\end{tabular}

Table 10: Shown Expected Sample Size of $\widetilde{\mathrm{A}}_{\mathrm{DS}}$ W.R.T. $\Delta$, U, and $\zeta$

\begin{tabular}{|c|c|c|c|c|c|c|c|c|c|c|}
\hline \multirow{3}{*}{$\mathrm{u}$} & \multirow[b]{3}{*}{$\mathrm{n}_{1}$} & \multirow[b]{3}{*}{$\Delta$} & \multirow{2}{*}{\multicolumn{8}{|c|}{$\zeta$}} \\
\hline & & & & & & & & & & \\
\hline & & & 0.25 & 0.50 & 0.75 & 1 & 1.50 & 1.25 & 1.75 & 2 \\
\hline & & 0.01 & 17.597 & 6.285 & 4.650 & 4.480 & 5.297 & 7.254 & 10.198 & 13.762 \\
\hline & 4 & 0.05 & 34.410 & 12.484 & 7.016 & 6.400 & 8.472 & 12.262 & 16.838 & 21.504 \\
\hline & & 0.1 & 42.132 & 18.080 & 9.776 & 8.801 & 11.644 & 16.293 & 21.400 & 26.234 \\
\hline & & 0.01 & 47.601 & 12.592 & 7.338 & 6.720 & 8.350 & 12.658 & 19.234 & 26.979 \\
\hline & 6 & 0.05 & 68.794 & 26.215 & 11.724 & 9.600 & 13.649 & 21.577 & 30.987 & 40.101 \\
\hline & & 0.1 & 74.270 & 36.504 & 16.494 & 13.200 & 18.709 & 28.215 & 38.317 & 47.280 \\
\hline & & 0.01 & 85.211 & 22.267 & 10.320 & 8.960 & 11.688 & 19.363 & 31.024 & 44.199 \\
\hline & & 0.1 & 102.995 & 60.006 & 24.450 & 17.601 & 26.577 & 42.377 & 58.413 & 71.645 \\
\hline & & 0.01 & 121.090 & 35.931 & 13.640 & 11.200 & 15.323 & 27.427 & 45.523 & 64.966 \\
\hline & 10 & 0.05 & 129.041 & 68.797 & 23.756 & 16.000 & 25.870 & 46.177 & 68.619 & 87.718 \\
\hline & & 0.1 & 129.783 & 87.173 & 33.624 & 22.001 & 35.231 & 58.579 & 81.064 & 98.232 \\
\hline & & 0.01 & 152.555 & 53.808 & 17.330 & 13.440 & 19.267 & 36.894 & 62.616 & 88.731 \\
\hline & 12 & 0.05 & 155.771 & 96.012 & 31.131 & 19.200 & 32.916 & 61.273 & 91.221 & 114.932 \\
\hline 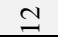 & & 0.1 & 155.960 & 116.678 & 43.999 & 26.399 & 44.654 & 76.629 & 105.719 & 126.205 \\
\hline
\end{tabular}

Table 11: Shown Expected Sample Size Proportion W.R.T. $\Delta, U, N_{1}$ and $\zeta$

\begin{tabular}{|c|c|c|c|c|c|c|c|c|c|c|}
\hline \multirow[b]{2}{*}{$\mathrm{u}$} & \multirow[b]{2}{*}{$\mathrm{n}_{1}$} & \multirow[b]{2}{*}{$\Delta$} & \multicolumn{8}{|c|}{$\zeta$} \\
\hline & & & 0.25 & 0.50 & 0.75 & 1 & 1.25 & 1.50 & 1.75 & 2 \\
\hline \multirow{14}{*}{12} & & 0.01 & 0.338 & 0.121 & 0.089 & 0.086148842 & 0.102 & 0.139 & 0.196 & 0.265 \\
\hline & 4 & 0.05 & 0.662 & 0.24 & 0.135 & 0.123082276 & 0.163 & 0.236 & 0.324 & 0.414 \\
\hline & & 0.1 & 0.81 & 0.348 & 0.188 & 0.169253764 & 0.224 & 0.313 & 0.412 & 0.504 \\
\hline & & 0.01 & 0.61 & 0.161 & 0.094 & 0.086154346 & 0.107 & 0.162 & 0.247 & 0.346 \\
\hline & 6 & 0.05 & 0.882 & 0.336 & 0.15 & 0.123079188 & 0.175 & 0.277 & 0.397 & 0.514 \\
\hline & & 0.1 & 0.952 & 0.468 & 0.211 & 0.169230694 & 0.24 & 0.362 & 0.491 & 0.606 \\
\hline & & 0.01 & 0.819 & 0.214 & 0.099 & 0.086153046 & 0.112 & 0.186 & 0.298 & 0.425 \\
\hline & & 0.1 & 0.99 & 0.577 & 0.235 & 0.169243129 & 0.256 & 0.407 & 0.562 & 0.689 \\
\hline & & 0.01 & 0.931 & 0.276 & 0.105 & 0.086154292 & 0.118 & 0.211 & 0.35 & 0.5 \\
\hline & 10 & 0.05 & 0.993 & 0.529 & 0.183 & 0.123077704 & 0.199 & 0.355 & 0.528 & 0.675 \\
\hline & & 0.1 & 0.998 & 0.671 & 0.259 & 0.169239908 & 0.271 & 0.451 & 0.624 & 0.756 \\
\hline & & 0.01 & 0.978 & 0.345 & 0.111 & 0.086152398 & 0.124 & 0.236 & 0.401 & 0.569 \\
\hline & 12 & 0.05 & 0.999 & 0.615 & 0.200 & 0.123075521 & 0.211 & 0.393 & 0.585 & 0.737 \\
\hline & & 0.1 & 1 & 0.748 & 0.282 & 0.169222587 & 0.286 & 0.491 & 0.678 & 0.809 \\
\hline
\end{tabular}

Table 12: Shown Percentage of Overall Sample Saved W.R.T. $\Delta, \mathrm{U}, \mathrm{N}_{1}$ and $\zeta$

\begin{tabular}{|c|c|c|c|c|c|c|c|c|c|c|}
\hline \multirow[b]{2}{*}{$\mathrm{u}$} & \multirow[b]{2}{*}{$\mathrm{n}_{1}$} & \multirow[b]{2}{*}{$\Delta$} & \multicolumn{8}{|c|}{$\zeta$} \\
\hline & & & 0.25 & 0.50 & 0.75 & 1 & 1.25 & 1.50 & 1.75 & 2 \\
\hline \multirow{14}{*}{12} & & 0.01 & 66.16 & 87.913 & 91.057 & 91.385115832 & 89.814 & 86.051 & 80.388 & 73.534 \\
\hline & 4 & 0.05 & 33.827 & 75.992 & 86.508 & 87.691772394 & 83.708 & 76.419 & 67.619 & 58.645 \\
\hline & & 0.1 & 18.977 & 65.231 & 81.2 & 83.07462357 & 77.608 & 68.667 & 58.845 & 49.55 \\
\hline & & 0.01 & 38.973 & 83.856 & 90.593 & 91.384565429 & 89.295 & 83.772 & 75.34 & 65.411 \\
\hline & 6 & 0.05 & 11.802 & 66.391 & 84.97 & 87.6920812 & 82.502 & 72.337 & 60.273 & 48.588 \\
\hline & & 0.1 & 4.782 & 53.2 & 78.854 & 83.076930636 & 76.014 & 63.826 & 50.876 & 39.384 \\
\hline & & 0.01 & 18.066 & 78.589 & 90.077 & 91.384695383 & 88.762 & 81.382 & 70.17 & 57.501 \\
\hline & & 0.1 & 0.966 & 42.302 & 76.49 & 83.075687055 & 74.445 & 59.253 & 43.834 & 31.11 \\
\hline & & 0.01 & 6.853 & 72.361 & 89.508 & 91.384570826 & 88.213 & 78.902 & 64.982 & 50.026 \\
\hline & 10 & 0.05 & 0.738 & 47.079 & 81.726 & 87.692229598 & 80.1 & 64.479 & 47.216 & 32.525 \\
\hline & & 0.1 & 0.167 & 32.944 & 74.135 & 83.076009249 & 72.899 & 54.939 & 37.643 & 24.437 \\
\hline & & 0.01 & 2.208 & 65.508 & 88.891 & 91.384760162 & 87.649 & 76.35 & 59.862 & 43.121 \\
\hline & 12 & 0.05 & 0.147 & 38.454 & 80.044 & 87.692447874 & 78.9 & 60.723 & 41.525 & 26.326 \\
\hline & & 0.1 & 0.026 & 25.206 & 71.795 & 83.077741289 & 71.376 & 50.879 & 32.231 & 19.099 \\
\hline
\end{tabular}

Table 13: Shown Probability of A Voiding Second Sample W.R.T. $\Delta, \mathrm{U}, \mathrm{N}_{1}$ and $\zeta$

\begin{tabular}{|c|c|c|c|c|c|c|c|c|c|}
\hline \multirow[b]{2}{*}{$\mathrm{n}_{1}$} & \multirow[b]{2}{*}{$\Delta$} & \multirow[b]{2}{*}{0.25} & \\
\hline & & & 0.50 & 0.75 & 1 & 1.25 & 1.50 & 1.75 & 2 \\
\hline \multirow{3}{*}{4} & 0.01 & 0.717 & 0.952 & 0.986 & 0.990005 & 0.973 & 0.932 & 0.871 & 0.797 \\
\hline & 0.05 & 0.366 & 0.823 & 0.937 & 0.949994 & 0.907 & 0.828 & 0.733 & 0.635 \\
\hline & 0.1 & 0.206 & 0.707 & 0.88 & 0.89997 & 0.841 & 0.744 & 0.637 & 0.537 \\
\hline \multirow{3}{*}{6} & 0.01 & 0.422 & 0.908 & 0.981 & 0.989999 & 0.967 & 0.908 & 0.816 & 0.709 \\
\hline & 0.05 & 0.128 & 0.719 & 0.921 & 0.949997 & 0.894 & 0.784 & 0.653 & 0.526 \\
\hline & 0.1 & 0.052 & 0.576 & 0.854 & 0.90000 & 0.823 & 0.691 & 0.551 & 0.427 \\
\hline \multirow{2}{*}{8} & 0.01 & 0.196 & 0.851 & 0.976 & 0.990000 & 0.962 & 0.882 & 0.76 & 0.623 \\
\hline & 0.05 & 0.035 & 0.612 & 0.903 & 0.949991 & 0.881 & 0.74 & 0.579 & 0.432 \\
\hline
\end{tabular}




\begin{tabular}{|c|c|c|c|c|c|c|c|c|c|}
\hline & 0.1 & 0.01 & 0.458 & 0.829 & 0.89998 & 0.806 & 0.642 & 0.475 & 0.337 \\
\hline & 0.01 & 0.045 & 0.721 & 0.936 & 0.836 & 0.603 & 0.381 & 0.225 & 0.129 \\
\hline \multirow{2}{*}{10} & 0.05 & $7.991 \mathrm{E}-3$ & 0.51 & 0.885 & 0.949999 & 0.868 & 0.699 & 0.512 & 0.352 \\
\hline & 0.1 & $1.807 \mathrm{E}-3$ & 0.357 & 0.803 & 0.899990 & 0.79 & 0.595 & 0.408 & 0.265 \\
\hline \multirow[t]{2}{*}{12} & 0.05 & $1.59 \mathrm{E}-3$ & 0.417 & 0.867 & 0.950002 & 0.855 & 0.658 & 0.45 & 0.285 \\
\hline & 0.1 & $2.771 \mathrm{E}-4$ & 0.273 & 0.778 & 0.900009 & 0.773 & 0.551 & 0.349 & 0.207 \\
\hline
\end{tabular}

\section{Conclusions}

From the above discussions it is obvious that by using guess point value one can improve the minimax estimator by using shrinkage technique. It can be noted that if the guess point $\alpha_{0}$ is very close to the true value of the parameter $\alpha$ (i.e.; $\zeta$ is approximate close to one), the proposed estimators perform better than the minimax estimator. If one has no confidence in the guessed value, then proposed preliminary test shrunken estimators can be suggested. We can safely use the proposed estimators for small sample size at the usual level of significance $\Delta$ and moderate value of shrunken weight factor $\Psi($.$) . The difficulty of obtaining samples because of$ the scarcity and high cost led researchers to use the double stage shrinkage estimators to reduce the sample size that we need and for achieving savings of the items in the sample and obtaining high efficiency estimators.

\section{Acknowledgments}

The authors wanted to provide thanks to the referees and to the Editor for constructive suggestions and valuable comments which resulted in the improvement of this article.

\section{References}

[1] Al-Joboori, A.N., (2010), Pre-Test Single and Double Stage Shrunken Estimators for the Mean of Normal Distribution with Known Variance, Baghdad Journal for Science, Vol.7(4), pp.14321442 .

[2] Al-Joboori, A.N., et al (2014), Single and Double Stage Shrinkage Estimators for the Normal Mean with the Variance Cases , International Journal of Statistics , Vol.(38),2,PP,1127 -1134.

[3] Aludaat 1, K.M., Alodat, M.T. and Alodat, T.T., (2008), Parameter Estimation of Burr Type X Distribution for Grouped Data, Applied Math. Sci., Vol. 2 (9), pp.415-423.

[4] Burr, I.W., (1942), Cumulative Frequency Distribution, Annals of Mathematical Statistics, Vol. (13), pp.215-232.

[5] Iman Makhdoom, (2011), Minimax Estimation of the Parameter of the Generalized Exponential Distribution, International Journal of Academic Research, Vol.3 (2), pp.515-527.

[6] Katti, S.K., (1962), Use of Some a Prior Knowledge in the Estimation of Means from Double Samples, Biometrics, Vol.18, pp.139147. http://dx.doi.org/10.2307/2527452.

[7] Kundu, D. and Raqab, M.Z., (2005), Generalized Rayleigh Distribution: Deferent Methods of Estimation, Computational Statistics and Data Analysis, Vol.49, pp.187-200. http://dx.doi.org/10.1016/j.csda.2004.05.008.

[8] Lanpong Li ,(2013), Minimax Estimation of Generalized Exponential Distribution under Square Log Error Loss and MLINEX Loss Function , Research Journal of Mathematics and Statistics, Vol.5(3),pp.24-27.

[9] Maha.A.Mohammed, (2011), Double Stage Shrinkage Estimator of Two Parameters Generalized Rayleigh Distribution, Education college journal AL-Mustansiriya University, Vol. (2), pp.566-573.

[10] Masoud Yarmohammadi and Hassan Pazira ,(2010),Minimax Estimation of the Parameter of the Burr Type Xii Distribution, Australian Journal of Basic and Applied Sciences, Vol.4(12), pp.66116622.

[11] M.Kamruj Jaman Bhuiyan ,et al,(2007) ,Minimax Estimation of the Parameter of the Rayleigh Distribution , Festschrift in honor Distinguished Professor Mir Masson Ali on the occasion of his retirement,pp.207-212.

[12] Raqab, M.Z., (1998), Order Statistics from the Burr Type X Model, Computers Mathematics and Applications, Vol.36, pp.111-120. http://dx.doi.org/10.1016/S0898-1221(98)00143-6.
[13] Raqab, M.Z. and Kundu, D., (2006), Burr Type X Distribution, Revisited, Journal of Probability and Statistical Sciences, Vol.4 (2), pp.179-193.

[14] Rodriguez, R.N., (1977), A Guide to Burr Type XII Distributions, Biometrika, Vol.64, pp.129-134. http://dx.doi.org/10.1093/biomet/64.1.129.

[15] Surles, J.G. and Padgett, W.J., (2001), Inference for Reliability and Stress-Strength for a Scaled Burr Type X Distribution, Lifetime Data Analysis, Vol.7, pp.187-200. http://dx.doi.org/10.1023/A:1011352923990.

[16] Surles, J.G. and Padgett, W.J., (2005), Some Properties of a Scaled Burr Type X Distribution, Journal of Statistical Planning and Inference, Vol.72, pp.271-280 http://dx.doi.org/10.1016/j.jspi.2003.10.003.

[17] Thompson, J.R., (1968), Some Shrinkage Techniques for Estimating the Mean, J. Amer. Statist. Assoc, Vol.63, pp.113-122.

[18] Waikar, V.B., Schuurmann, F.J. and Raghunathar, T.E., (1984), On a Two-Stage Shrinkage Testimator of the Mean of a Normal Distribution, Commum. Statist-Theory. Meth. A, Vol.13 (15), pp.19011913. http://dx.doi.org/10.1080/03610928408828802.

[19] Wingo, D.R., (1993), Maximum Likelihood Methods for fitting the Burr Type XII Distribution to Multiply (Progressively) Censored Life Test Data, Metrika, Vol. 40, pp.203-210. http://dx.doi.org/10.1007/BF02613681. 